\title{
Spectrum Sensing in Public Safety Applications: The 2-D LAD ACC Method
}

\author{
Johanna Vartiainen, Janne Lehtomäki, Timo Bräysy \\ University of Oulu \\ Centre for Wireless Communications (CWC) \\ P.O. Box 4500, FIN-90014 University of Oulu, FINLAND
}

\author{
Kenta Umebayashi \\ Tokyo University of Agriculture \\ and Technology (TUAT) \\ Koganei-Shi, 184-8588 JAPAN
}

\begin{abstract}
Public safety communications (PSC) are invaluable in safety critical scenarios such as floods or earthquakes when commercial communication systems fall down. Cognitive radio (CR) approach that is independent of other communication systems provides flexibility and certainty. One of the key issues is to reliably find unused radio channels also in the situations when there is no a priori information about possible other users. In this paper, we propose using the two-dimensional localization algorithm based on double-thresholding with adjacent cluster combining (2-D LAD ACC) method for detecting free channels. It is low-complex, blind and able to operate in any frequency area. This paper applies the 2-D LAD ACC method for realworld channel measurement data for the first time. The indoor measurements at WLAN bands verify that the 2-D LAD ACC method is able to operate in the real-life situation.
\end{abstract}

\section{INTRODUCTION}

Wireless public safety communications (PSC) are used by emergency service organizations to prevent or respond to incidents that harm or endanger people or property [1], [2]. In its fullest extent, PSC can involve a unique application of a set of principles, a combination of technologies, and integration of processes to provide assured communications support for deployed emergency responders. In Europe, 380$400 \mathrm{MHz}$ frequencies are often used for the narrowband public safety communications utilizing terrestrial trunked radio (TETRA) or similar technology. The problem is that current spectrum allocations do not support the wide scale use of wide bandwidth applications, such as live video transmission in rescue operations. Additional spectrum for PSC could be available, for example, from the TV-band white spaces ("digital dividend") or in industrial, scientific and medical (ISM) bands which are around $2.4 \mathrm{GHz}$ and $5.8 \mathrm{GHz}$.

Here we are focusing on cognitive radio (CR) based dynamic spectrum access (DSA) use on safety critical scenarios. For example, if the commercial communications infrastructure (or part of it) falls down as a result of the catastrophic event such as major flood or earthquake, the mobile PSC infrastructure could use CR techniques for finding and managing channels that are unused.

DSA describes the technique where prior to transmitting, a CR attempts to detect the presence of other radio systems operating in the bands and avoids operating on frequencies that could cause interference with those systems [3], [4], [5].

This research was funded by EU FP7 project Euler (FP7-SEC-218133).
In a public safety scenario, it is natural to assume that several organizations might be interested to use the same spectrum, and therefore it is important to try to share spectrum fairly, and not to interfere other operators, both other PSC organizations and possibly some civilian networks still operational.

The key issue and the first step in DSA is to locate the available spectrum slots both in frequency and in time. This can be done using signal detection methods to decide if a signal or signals are present or not, i.e., if the investigated frequency band is occupied or available. Further steps in full DSA implementation would be to propose an autonomous spectrum management methodology. Cognitive techniques for finding free spectrum for PSC operation has been considered, for example, in [6], [7].

Finding free spectrum for a transmission is a demanding task in PSC. One of the biggest challenges is that there are several types of signal formats or several organizations PSC. For example, in ISM band, there are signals like wireless local area networks (WLAN), bluetooth, fixed wireless access (FWA), etc. In addition, the detected signals can be either secondary or primary user signals with only some or even none a priori information. Thus, blind and flexible signal detection techniques are required. It is also beneficial if the detection method is low-complex and capable of autonomous operation if a connection to the outside world is lost.

The two-dimensional localization algorithm based on double-thresholding with adjacent cluster combining (2-D LAD ACC) method could be a key in the DSA in PSC. The 2-D LAD ACC method is an iterative method for detecting signals blindly [8], [9]. It can be considered constant false alarm rate (CFAR) type energy detector. The 2-D LAD ACC method is robust to noise level, used signal and modulation type and frequency-shifting, and it can be used all over the spectrum. The only requirement is that the signal(s) should be narrow with respect to the considered bandwidth. The versatile 2-D LAD ACC method can be used, for example, in spectrum sensing in cognitive radios [8] or interference suppression. Its overall computational complexity is low, of the order of $N \log _{2} N$, which enables real-time implementation [9]. Thus, the 2-D LAD ACC method is very suitable for being used for public safety applications. This paper demonstrates the performance of the 2-D LAD ACC method for real-world ISM band. To the best of the authors' knowledge, this is the 


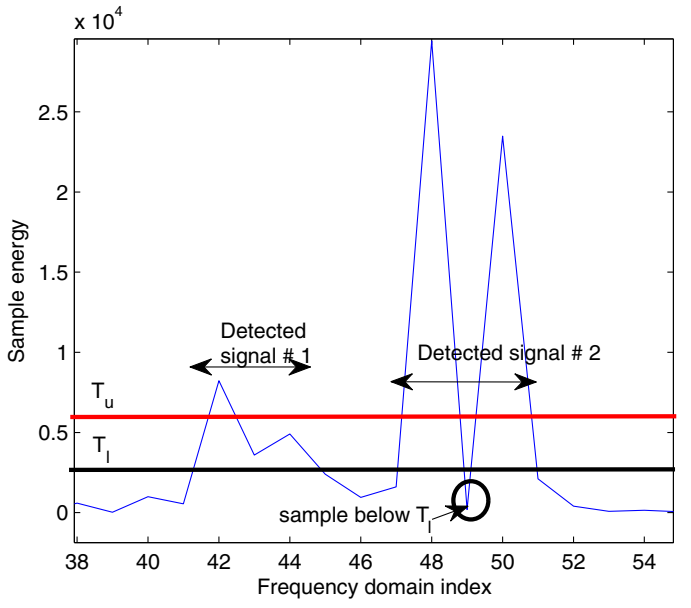

Fig. 1. The LAD and LAD ACC methods. $T_{u}$ denotes the upper threshold as $T_{l}$ denotes the lower threshold.

TABLE I

THE FCME ALGORITHM.

Preliminary:

Select threshold parameter $T_{C M E}[10]$

Rearrange squared samples in ascending order according to sample energy Select $m=10 \%$ smallest samples to form the initial set $Q$ Iterative calculation:

Step 1: Calculate threshold $T_{h}=T_{C M E} * \bar{Q}$, where ${ }^{-}$is the sample mean Step 2: Add samples $<T_{h}$ to the set $Q$

Repeat steps 1 and 2 until there are no new samples below the threshold Result:

Samples above the threshold are signal samples

first time when the 2-D LAD method has been demonstrated in practice. Even though the indoor measurements have been performed in WLAN bands at $2.4 \mathrm{GHz}$, the results can be generalized to other radio channels.

\section{THE 2-D LAD ACC METHOD}

The 2-D LAD ACC method consists of three parts: the LAD method, LAD ACC and 2-D LAD processing. The LAD method is based on the usage of two thresholds. The thresholds are usually calculated with the forward consecutive mean excision (FCME) algorithm [10] (Table I) which is run twice with two different threshold parameters to have two thresholds, or set manually. After the threshold setting, the LAD method groups the adjacent samples above the lower threshold into the same group, a cluster. If at least the largest element of a cluster exceeds the upper threshold, the cluster is deemed to be caused by a signal and it is accepted. Instead, if the largest sample is below the upper threshold, the cluster corresponds to the noise (Fig. 1, signal \#1).

In the LAD ACC processing, an extra condition after the LAD processing is used. That is, if accepted clusters are separated only by at most $n$ (typically $n=1-3$ ) samples below the lower threshold, it is deemed that there is only one signal (Fig. 1, signal \#2). This means that when using the LAD ACC processing, two signals can be separated if there is $n+1$ or more adjacent samples between the signals that are

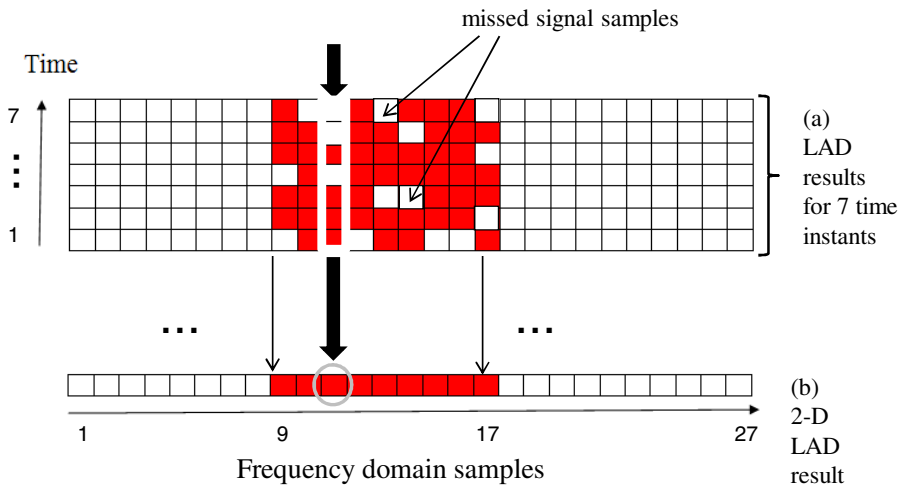

Fig. 2. The 2-D LAD processing. One example output. Here $p=4$ and $r=7$ samples. [9]

below the lower threshold.

The 2-D LAD processing [8] coincides with binary detection in radar systems. After the LAD ACC (or LAD) in the frequency domain, time domain processing is performed. That is, $r$ consecutive time intervals are considered to decide if the samples are caused by a signal or not. If a specific frequency domain sample is reported to belong to the signal at least $p$ times out of $r$ time instants, that frequency domain sample is deemed to belong to the signal (Fig. 2). Optimal selection of parameters $n, p$ and $r$ depends on application. For more details, see [9] and references therein.

\section{MEASUREMENTS}

\section{A. Measurement Environment}

In the measurements, we monitored the whole $2.4 \mathrm{GHz}$ ISM-band. There were three laptops and one access point (AP). The AP was Linksys WRT54G. The laptop 1 generated large amounts of traffic by using the Bwping program and slept for a given time period before starting the next cycle. Channel 1 was used to the transmission. For power measurement (the spectrogram) we used a laptop 2 equipped with the FLUKE Networks AnalyzeAir ${ }^{\mathrm{TM}}$ Wi-Fi Spectrum Analyzer. The Spectrum Analyzer was set to use the maximum RF power seen during the measurement interval based on several FFTs for each spectrogram bin value, in each sweep. Sweep time was one second. The laptop 2 recorded the spectrogram outputs automatically by using script written using AutoIt. The outputs were processed with MATLAB program, which performed the 2-D LAD ACC method. For reference purpose, a 3rd laptop was located close to the Spectrum Analyzer laptop and it was capturing packets by using Wireshark and the laptop WLAN card was set the "monitor mode" to capture all WLAN traffic in the air. By this arrangement, we compare the sensing results by Spectrum Analyzer with the packet reception performance of a typical WLAN card.

In the 2-D LAD ACC method, we used three parameter modes called sensitive, strict and adaptive. The sensitive and strict mode used fixed, manually set thresholds, as the adaptive mode calculated the thresholds adaptively using the FCME algorithm. In the sensitive mode, the thresholds are relatively 
TABLE II

USED PARAMETER MODES IN THE 2-D LAD ACC METHOD.

\begin{tabular}{l||l|l|l|l|l} 
Parameters & Upper threshold & Lower threshold & $n$ & $p$ & $r$ \\
\hline Sensitive & $-88 \mathrm{dBm}$ & $-96 \mathrm{dBm}$ & 3 & 1 & 5 \\
Strict & $-80 \mathrm{dBm}$ & $-90 \mathrm{dBm}$ & 3 & 4 & 6 \\
Adaptive & calculated & calculated & 3 & 1 & 5 \\
& using FCME & using FCME & & & \\
& $T_{C M E}=13.81$ & $T_{C M E}=2.66$ & & &
\end{tabular}

low, as in the strict mode, the thresholds are relatively high. The parameters in each mode are presented in Table II.

Here we have defined that the height (transmission time, or signal duration) and width (frequencies) of the transmitted signal constitutes the signal area. Thus, the signal is detected perfectly if both the transmission time and frequencies are estimated properly. False alarms were defined to be detected signal samples outside the nominal WLAN channel 1 (signal area).

\section{B. EMC Measurements}

In the EMC measurements, there were no interference or other WLAN signals. AP was configured to the pure B-mode and the beacon interval was set to 10 seconds. Different signal-to-noise ratio (SNR) levels were obtained by adding attenuating blocks around the transmitter and the AP. We made measurements with three different SNR levels $( \pm 4 \mathrm{~dB})$ based on $500(\mathrm{SNR}=50$ and $20 \mathrm{~dB})$ or $60(\mathrm{SNR}=10 \mathrm{~dB})$ transmitted cycles. One cycle duration was about one minute $(30 \mathrm{sec}$. transmission and $30 \mathrm{sec}$. silence). Both time and frequency detection were considered. The channel was not line-of-sight except in the case of SNR=50 dB.

The mean signal duration and average estimated signal durations are presented in Table III. It can be seen that the 2-D LAD ACC method was able to estimate the signal duration very well with all parameter modes (sensitive, strict and adaptive).

Results for detected signal area are presented in Table IV. The 2-D LAD ACC performed very well when SNR was 20 $\mathrm{dB}$ or more. For example, when $\mathrm{SNR}=50 \mathrm{~dB}$ and adaptive parameters were used, the 2-D LAD ACC method detected, on average, $99.9 \%$ of the signal area. In the best/worst cycle, $100 \% / 95.5 \%$ of the signal area was detected, respectively. Note, that low detection performances were mainly because missing the sidelobes. However, depending on application, detecting sidelobes is not necessarily needed.

The false alarm rates $\left(P_{F A}\right)$ are presented in Table V. At $\mathrm{SNR}=50 \mathrm{~dB}$, high false alarms were caused by the sidelobes of strong signals extending beyond the nominal WLAN channel 1. At most, sidelobes extended to almost $2438 \mathrm{MHz}$.

\section{Office Measurements}

In the measurements performed in a typical office environment, we used three laptops and one AP. The AP was configured to use the pure-G mode and the beacon interval was increased to 2 seconds to reduce the amount of beacon traffic. One cycle duration was about $100 \mathrm{sec}$. (40 sec. transmission and $60 \mathrm{sec}$. silence). The AP was located close to
TABLE III

EMC MEASUREMENTS WITH NO INTERFERENCE. THE MEAN SIGNAL DURATION AND AVERAGE ESTIMATED SIGNAL DURATIONS BY THE 2-D LAD ACC METHOD.

\begin{tabular}{l||l|l|l|l|l|l}
\multicolumn{1}{l||}{} & \multicolumn{6}{c}{ Estimated signal duration [sec.] } \\
Signal & \multicolumn{5}{c}{ SNR=50 dB } & \multicolumn{3}{c}{ SNR=20 dB } \\
& Sensitive & Strict & Adapt. & Sensitive & Strict & Adapt. \\
\hline 31.31 & 32.64 & 30.73 & 31.06 & 31.97 & 30.56 & 31.75
\end{tabular}

TABLE IV

EMC MEASUREMENTS WITH NO INTERFERENCE. DETECTION PERFORMANCE OF THE 2-D LAD ACC METHOD.

\begin{tabular}{l||l|l|l}
\multicolumn{1}{c||}{} & \multicolumn{3}{c}{ Detected signal area [\%]: best/worst/on average } \\
Parameters & SNR=50 dB & SNR=20 dB & SNR=10 dB \\
\hline Sensitive & $100 / 95.5 / 99.9$ & $89.6 / 69.5 / 75.9$ & $53.2 / 0 / 11.9$ \\
Strict & $99.9 / 92.1 / 97.1$ & $69.4 / 50.5 / 56.8$ & $0 / 0 / 0$ \\
Adaptive & $100 / 95.5 / 99.9$ & $93.5 / 71.9 / 78.1$ & $46.5 / 0 / 3.0$ \\
\hline Laptop & 100 & 70 & 50
\end{tabular}

TABLE V

EMC MeAsurements With No InTERference. $P_{F A}$ OF the 2-D LAD ACC METHOD.

\begin{tabular}{l||l|l|l}
\multicolumn{1}{c||}{} & \multicolumn{3}{c}{$P_{F A}[\%]$} \\
Parameters & SNR=50 dB & SNR=20 dB & SNR=10 dB \\
\hline Sensitive & 7.5 & 0.8 & 0.1 \\
Strict & 0.8 & 0 & $-^{*}$ \\
Adaptive & 9.7 & 0.9 & 0.05
\end{tabular}

* no signals found

TABLE VI

OFFICE MEASUREMENTS. DISTANCES [M] BETWEEN MEASUREMENT POINTS (MP), LAPTOP (LT) 2 AND ACCESS POINT (AP).

\begin{tabular}{l||l|l|l|l} 
& MP \#1 & MP \#2 & MP \#3 & MP \#4 \\
\hline LT 2 & 4.8 & 13.3 & 19.6 & 32.3 \\
AP & 34.2 & 20 & 13.4 & 0.5
\end{tabular}

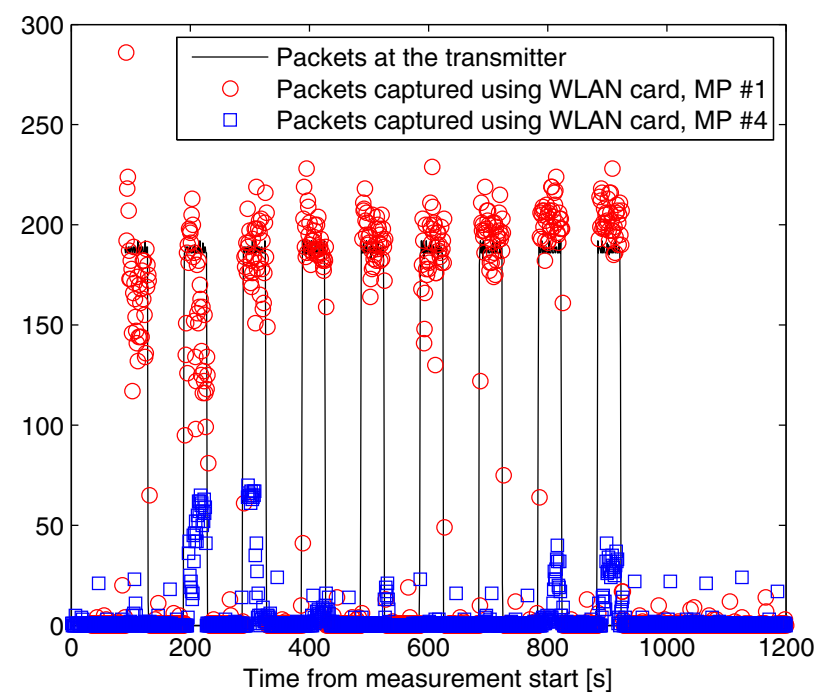

Fig. 4. Transmitter at MPs \#1 and \#4, Wireshark IO-results at the transmitter and the receiver (sensing laptop).

the measurement point (MP) \#4. The laptop 1 was used as a transmitter and it was moved between MPs \#1-4 selected to obtain different signal levels (Fig. 3). The distances between 


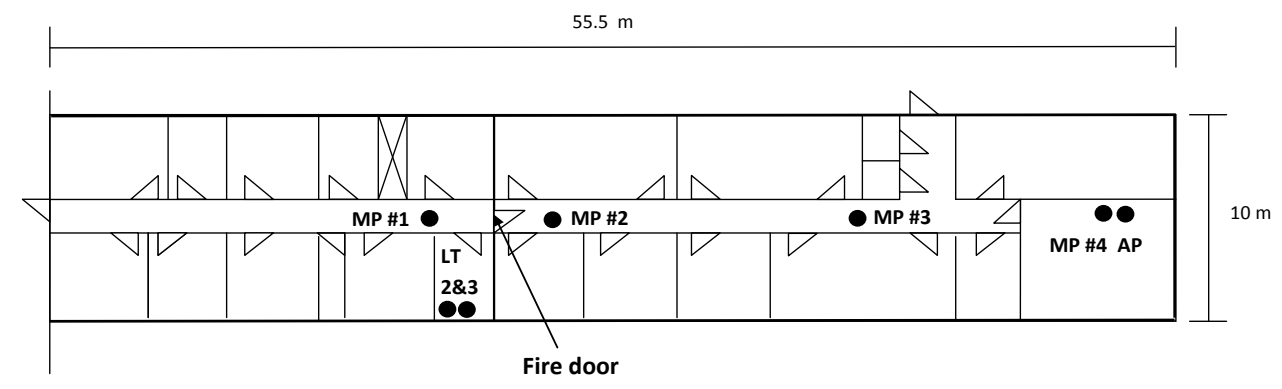

Fig. 3. The plan drawing. Measurement points (MP), access point (AP) and laptops (LT).

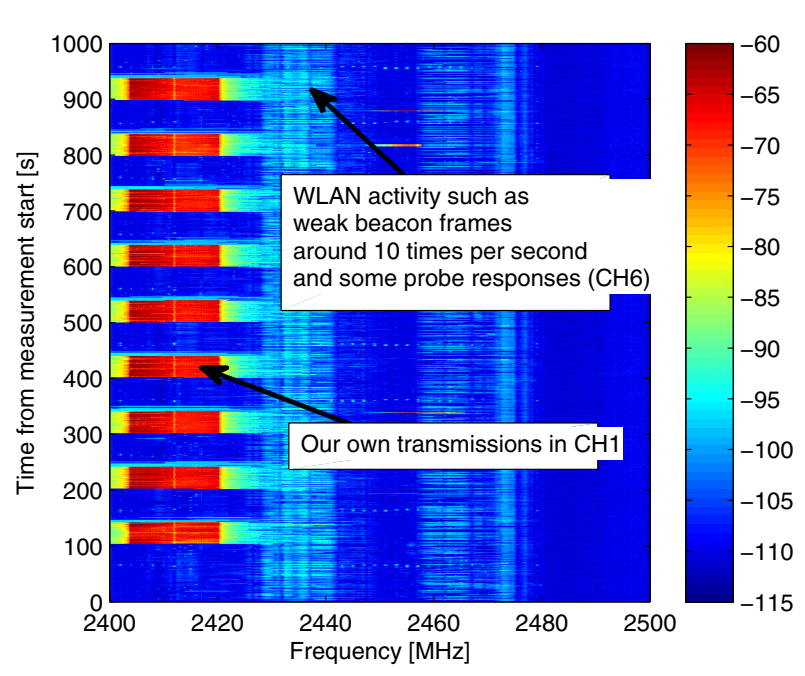

Fig. 5. Transmitter at MP \#1, original spectrogram, resolution bandwidth $156 \mathrm{kHz}$.

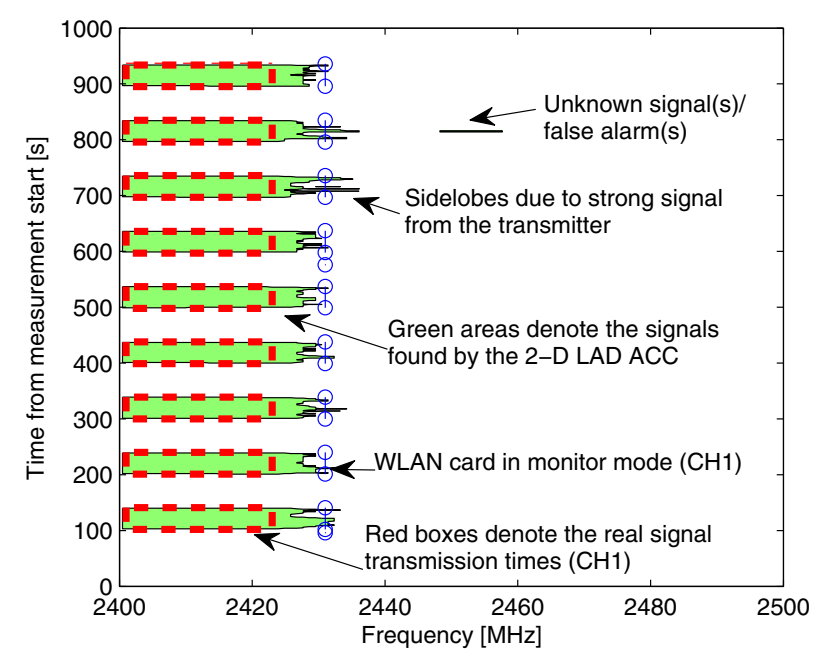

Fig. 6. Transmitter at MP \#1, processed spectrogram, 2-D LAD ACC with strict parameters, resolution bandwidth $937 \mathrm{kHz}$.

AP, MPs and laptop 2 are presented in Table VI. We recorded the packet transmission times in the laptop 1 by using the
Wireshark packet analyzer. In the 2-D LAD ACC method sensitive and strict parameters were used.

Fig. 4 presents the number of transmitted and captured packets in MPs \#1 and 4. In MP \#1, the laptop 3 captured $98.2 \%$ of the packets due to strong signal strength. In the MP $\# 4$, the laptop 3 captured only $6 \%$ of the packets. This is because AP and MP \#4 were located close to each other. In MPs \#2 and \#3, the laptop 3 captured $46.4 \%$ and $43.4 \%$ of the packets, respectively.

Figs. 5 - 10 present results from different MPs. In the processed spectrograms, the "real" transmission times (red boxes) were generated from the packet transmission times. The results of the 2-D LAD ACC method are the "found" signals (green areas). In Figs. 5-7, the transmitter was at MP $\# 1$. Here, SNR is about $40 \mathrm{~dB}$. The sidelobes are due to the strong signal from the transmitter. Probability of detection is good with both strict and sensitive parameters.

Figs. 8 and 9 present the results when the transmitter was at $\mathrm{MP} \# 2$. Now, $\mathrm{SNR}=30 \mathrm{~dB}$. As can be seen, the 2-D LAD ACC method performed well with strict parameters. However, some sidelobes were missing. The time domain detection (transmission time estimation) was very good. Close to 2460 $\mathrm{MHz}$, there was an unknown signal which was also detected. The 2-D LAD ACC method with sensitive parameters detected all transmitted signals. As before, also activities in channel 6 were detected. Between the transmitted cycles there were some detections, that may be caused by probe requests, responses and beacons.

When the transmitter was at MP \#3, SNR=20 dB. The 2-D LAD ACC method with strict parameters detected something in each cycle (Fig. 10). Sensitive parameters performed better, but even there the sidelobes were not detected. At MP \#4, AP was close to MP and SNR=13 dB. The 2-D LAD method with strict parameters failed to detect signals. The result was expected, because in the strict mode, the thresholds are high. Instead, sensitive parameters performed better. However, the detected bandwidths were narrower than the real ones.

\section{CONCLUSIONS}

In this paper, we have discussed spectrum sensing in public safety communications. The 2-D LAD ACC method which is low-complex, blind and able to operate in any frequency area was proposed to be used for signal detection in public safety 


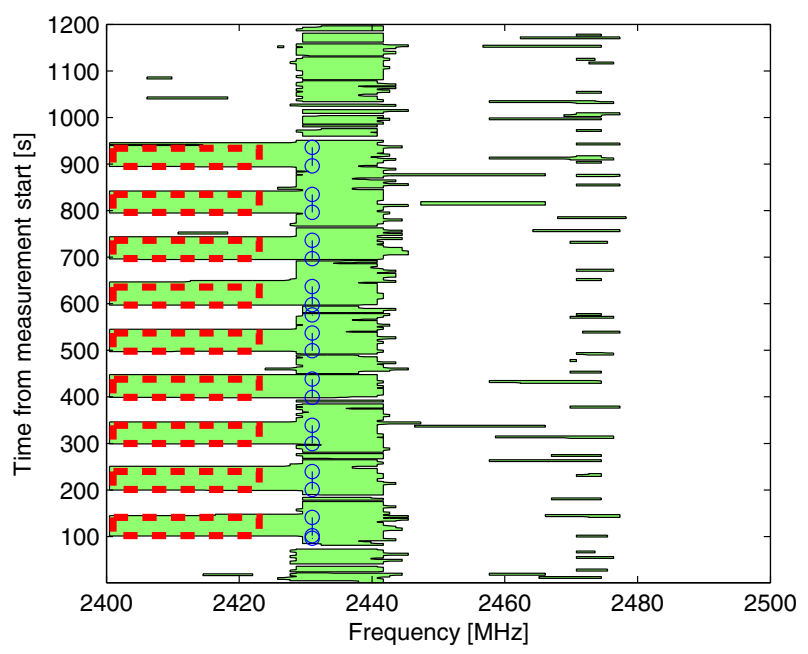

Fig. 7. Transmitter at MP \#1, processed spectrogram, 2-D LAD ACC with sensitive parameters, resolution bandwidth $937 \mathrm{kHz}$.

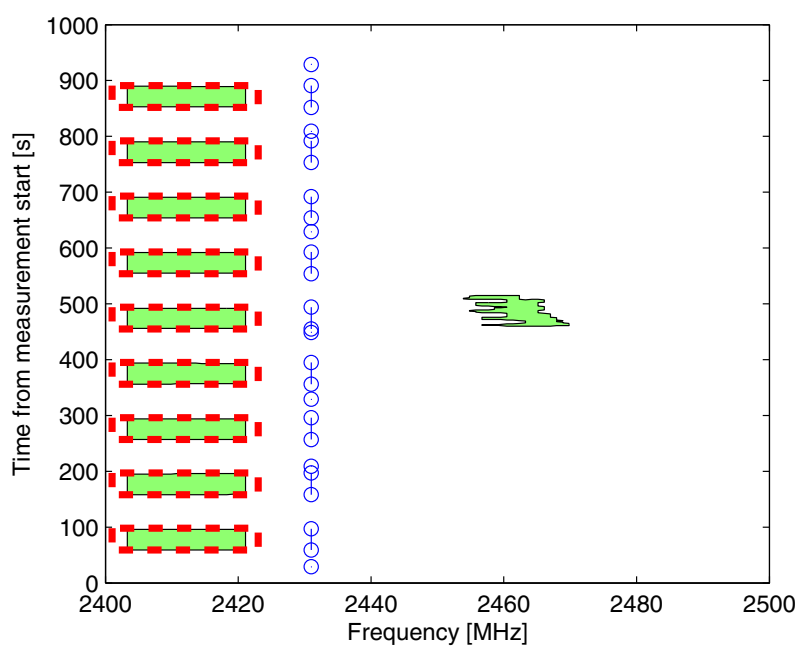

Fig. 8. Transmitter at MP \#2, processed spectrogram, 2-D LAD ACC with strict parameters, resolution bandwidth $937 \mathrm{kHz}$.

applications. The performance of the method was demonstrated in WLAN bands. Indoor measurements were performed both in EMC room and in a typical office environment. It can be concluded that the 2-D LAD ACC method had good performance.

\section{REFERENCES}

[1] H. Borgonjen, "Dynamic spectrum management and cognitive radio for the public safety," in 4th International Conference on Information Systems for Crisis Response and Management ISCRAM, Jun. 2009.

[2] FCC, "Public safety and homeland security bureau," 2011, http://publicsafety.fcc.gov/pshs/clearinghouse/core-concepts/index.htm.

[3] J. Mitola III and G. Q. Maguire Jr., "Cognitive radio: making software radios more personal," IEEE Personal Communications, vol. 6, no. 4, pp. 13-18, 1999.

[4] S. Haykin, "Cognitive radio: Brain-empowered wireless communications," IEEE Journal in Selected Areas in Comm, vol. 23, no. 2, pp. 201-220, Feb. 2005.

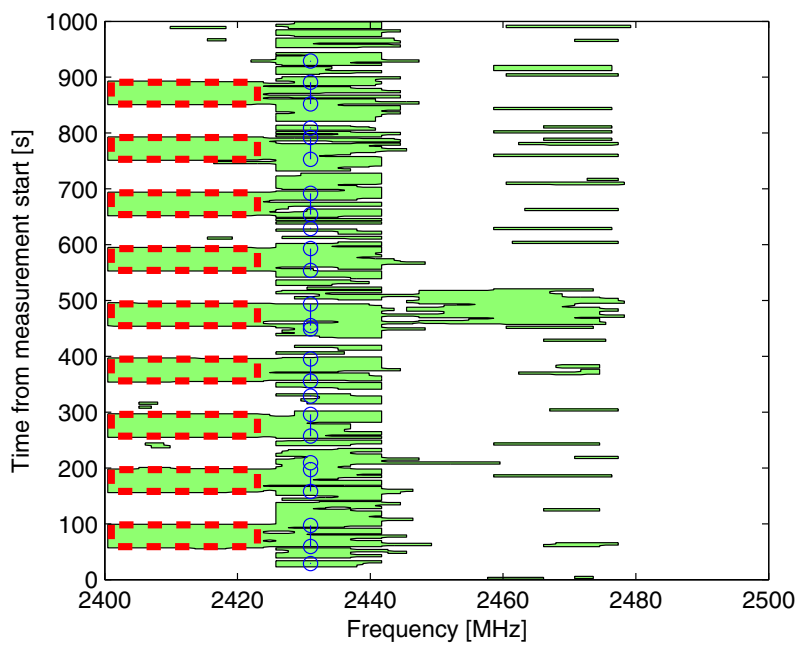

Fig. 9. Transmitter at MP \#2, processed spectrogram, 2-D LAD ACC with sensitive parameters, resolution bandwidth $937 \mathrm{kHz}$.

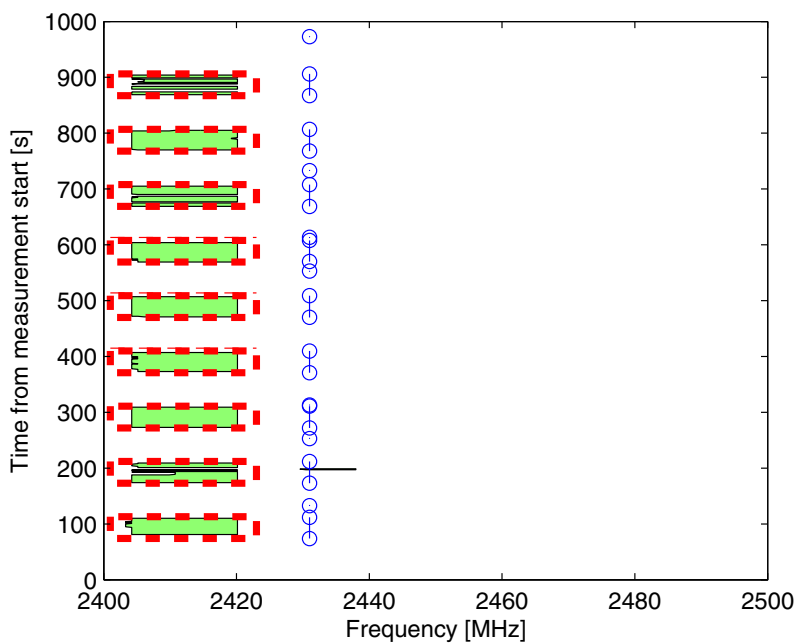

Fig. 10. Transmitter at MP \#3, processed spectrogram, 2-D LAD ACC with strict parameters, resolution bandwidth $937 \mathrm{kHz}$.

[5] T. Yucek and H. Arslan, "A survey of spectrum sensing algorithms for cognitive radio applications," IEEE Communications Surveys and Tutorials, vol. 11, no. 1, 2009.

[6] G. Feng et al., "Cognitive radio: From spectrum sharing to adaptive learning and reconfiguration," in IEEE Aerospace Conference, 2008, pp. $1-10$.

[7] T. Bräysy et al., "Cognitive techniques for finding spectrum for public safety services," in Military Communications and Information Systems Technology Week, Wrocklaw, Poland, Sep. 2010.

[8] J. Vartiainen, J. J. Lehtomäki, H. Saarnisaari, and M. Juntti, "Twodimensional signal localization algorithm for spectrum sensing," IEICE Transactions on Communications, vol. E93-B, no. 11, pp. 3129-3136, Nov. 2010

[9] J. Vartiainen, Concentrated Signal Extraction Using Consecutive Mean Excision Algorithms, Ph.D. thesis, Acta Univ Oul Technica C 368. Faculty of Technology, University of Oulu, Finland, Nov. 2010, http: //herkules.oulu.fi/isbn9789514263491/.

[10] H. Saarnisaari, P. Henttu, and M. Juntti, "Iterative multidimensional impulse detectors for communications based on the classical diagnostic methods," IEEE Transactions on Communications, vol. 53, no. 3, pp. 395-398, March 2005. 